\title{
HIV hides in haematopoietic stem cells
}

Although current treatments for HIV/AIDS seek to manage the disease, the ultimate aim of any therapy is complete eradication of the virus. Unfortunately, full eradication of HIV is challenging, as the virus establishes latent reservoirs in resting $\mathrm{CD}^{+} \mathrm{T}$ cells. Now, Carter et al. show that HIV can infect haematopoietic stem cells (HSCs); thus, the reservoir of HIV is even more persistent than previously thought.

Previous reports had suggested that HSCs are resistant to infection by HIV, but known viral reservoirs cannot fully account for the disease progression of AIDS, indicating that there are other reservoirs of latent infection. A study from the authors, published in 2010, suggested that HIV can infect haematopoietic progenitor cells (HPCs), but the assays used could not differentiate HSCs

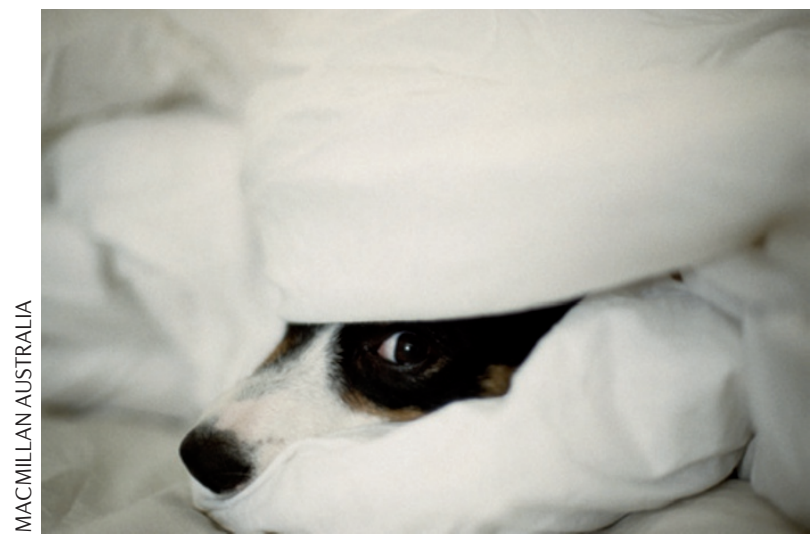

from other cell types within the HPC population. Now, using a multilineage engraftment experiment (that is, the ability of haematopoietic cells engrafted into sublethally irradiated mice to form multiple blood lineages and persist for more than 4-6 weeks, which is the definitive assay for stem cell potential), Carter et al. show that HIV can indeed infect HSCs.

They began the current study by investigating whether the cellular receptor that HIV uses to enter cells influences the type of HPCs that become infected. HIV enters cells through the interaction of the HIV envelope protein (Env) with the CD4 receptor and either of the chemokine co-receptors CXCR4 or CCR5.

The authors generated replicationdeficient, non-toxic HIV in a system in which they could vary the tropism of the Env protein. In addition, they tagged the HIV genome with GFP under a constitutive promoter, allowing the use of flow cytometry to isolate infected cells. They found that HIV particles containing a CXCR4-tropic Env protein could infect cells that have a CD $34^{\text {hi }} \mathrm{CD} 133^{+}$ phenotype, which is associated with multipotency. By contrast, using the CCR5-tropic Env protein, the virus could only efficiently infect the more differentiated $\mathrm{CD} 34^{\text {low }} \mathrm{CD} 133^{-}$cells. Next, the authors used colony formation assays, in which progenitor cells differentiate in vitro into colonies that are representative of the lineages that can form in vivo, to show that cells infected by CXCR4-tropic HIV can form colonies that are derived from immature HPCs, including HSCs. By contrast, CCR5-tropic HIV-infected cells formed very few colonies.

To confirm that CXCR4-tropic HIV can infect HSCs, the authors performed the multilineage engraftment experiment, introducing human HPCs infected with CXCR4-tropic HIV into irradiated mice. Humanderived, $\mathrm{GFP}^{+}$lymphoid and myeloid cells were detected in the blood of the mice up to 20 weeks after transplantation. This indicates that the original HIV-infected cells were indeed HSCs.

Thus, treatments to cure HIV will need to eradicate the reservoir of latent HIV in slow-dividing HSCs. However, as active infection with HIV causes HSCs to undergo apoptosis, it is possible that therapies to activate HSCs may be effective in reducing the HIV reservoir, in combination with standard antiretroviral therapy.

Joanna E. Huddleston

ORIGINAL RESEARCH PAPER Carter, C. et al. HIV-1 utilizes the CXCR4 chemokine receptor to infect multipotent hematopoietic stem and progenitor cells. Cell Host Microbe 9, 223-234 (2011)

FURTHER READING Carter, C. et al. HIV-1 infects multipotent progenitor cells causing cell death and establishing latent cellular reservoirs. Nature Med. 16, 446-452 (2010) 